\title{
COORDINATE AND MOMENTUM REPRESENTATIONS IN QUANTUM MEGHANIGS
}

\author{
D. F. LAWDEN
}

(Received 7 October 1966)

\section{Introduction}

On the basis of physical principles having a very general nature, A. Landé $[1]$ has demonstrated that the mathematical structure of quantum mechanics can be derived without having recourse to the introduction of special assumptions of an ad hoc type (such as commutation rules governing canonical observables) which are not immediately suggested by our knowledge of the physical world, but which have simply originated as rules which mathematical physicists have discovered by past experience to yield conclusions in conformity with experiment.

In particular, he establishes that the relationship between two representations of the state of a physical system must necessarily be of unitary type, i.e. if the sets of observables being employed as bases for the representations have discrete spectra, so that the system state is specified by vectors having components $\alpha_{i}, \alpha_{i}^{\prime}(i=1,2, \cdots)$ in the two representations, then

$$
\begin{aligned}
& \alpha_{i}^{\prime}=\sum_{j} u_{i j} \alpha_{j}, \\
& \alpha_{j}=\sum_{i} u_{i j}^{*} \alpha_{i}^{\prime},
\end{aligned}
$$

where $u_{i j}$ are the elements of a unitary matrix and (2) is the transformation inverse to (1), (asterisks indicate that the conjugate complex quantity is to be taken).

Landé also indicates that the well-known fundamental relationship between the coordinate and momentum representations of a particle's state, from which all other representations of the states of physical systems (ignoring spin observables) may be considered to be derived, follows by application of the special principle of relativity when this is supplemented by the assumption that the relationship between these two representations is unitary in character. The purpose of this note is to perform this derivation of the form of this relationship in a comparatively rigorous manner. 


\section{Derivation of the relationship}

Let $x_{i}(i=1,2,3)$ be the coordinates of the particle relative to an inertial frame $S$ and let $p_{i}(i=1,2,3)$ be the corresponding components of its linear momentum. Then, if $\psi\left(x_{1}, x_{2}, x_{3}\right)=\psi\left(x_{i}\right)$ is the wave function describing a particular state $A$ of the particle when the coordinate representation is being employed and if $\phi\left(p_{1}, p_{2}, p_{3}\right)=\phi\left(p_{i}\right)$ is the wave function describing the same state in the momentum representation, we shall assume that

$$
\begin{aligned}
\phi\left(p_{i}\right) & =\int u\left(p_{i}, x_{i}\right) \psi\left(x_{i}\right) d x, \\
\psi\left(x_{i}\right) & =\int u^{*}\left(p_{i}, x_{i}\right) \phi\left(p_{i}\right) d p,
\end{aligned}
$$

determine the form of the unitary transformation (1), (2), appropriate to the case when the observables of the basis possess continuous spectra. In equations (3) and (4), $d x=d x_{1} d x_{2} d x_{3}, d p=d p_{1} d p_{2} d p_{3}$ and the integrations extend over the full range $(-\infty, \infty)$.

In conformity with the special principle of relativity, we shall assume that the form of this relationship is independent of the inertial frame being employed, i.e. the form of the kernel $u$ does not alter if we transform from one inertial frame to another. Consider, therefore, a second inertial frame $S^{\prime}$ relative to which the particles coordinates are $x_{i}^{\prime}$ and its momentum components are $p_{i}^{\prime}$. Then, we have the orthogonal transformation equations

$$
\begin{aligned}
x_{i}^{\prime} & =\sum_{j=1}^{3} a_{i j} x_{j}+b_{i}, \\
p_{i}^{\prime} & =\sum_{j=1}^{3} a_{i j} p_{j}+c_{i},
\end{aligned}
$$

where the coefficients $a_{i j}$ are determined by the inclinations of the axes of $S^{\prime}$ to the axes of $S$ and satisfy the well-known conditions on the elements of an orthogonal matrix. It should here be noted that, although we are appealing to the special principle of relativity, our object is to develop, not Dirac's relativistic theory, but the classical theory of quantum mechanics. In the same way that the fundamental equations of classical Newtonian mechanics are covariant with respect to a Galilean transformation and, as a consequence, are consistent with the special principle of relativity, we are assuming that the classical theory of quantum mechanics exhibits a similar covariance with respect to this type of transformation. The equations (5) and (6) accordingly represent, not a Lorentz transformation, but a Galilean transformation referring to the particular time instant under consideration.

In the $x^{\prime}$-representation, let the state $A$ be determined by a wave 
function $\psi^{\prime}\left(x_{i}^{\prime}\right)$ and, in the $p^{\prime}$-representation, by a wave function $\phi^{\prime}\left(p_{i}^{\prime}\right)$. Then, the probability of finding the particle in the neighbourhood of a given point must be the same whether calculated in the $S$-frame or the $S^{\prime}$-frame and hence

$$
|\psi|=\left|\psi^{\prime}\right|
$$

Similarly,

$$
|\phi|=\left|\phi^{\prime}\right|
$$

Hence we can write

$$
\psi^{\prime}=\psi e^{i \alpha}, \quad \phi^{\prime}=\phi e^{\iota \beta},
$$

where

$$
\begin{aligned}
& \alpha=\alpha\left(x_{i}, a_{j k}, b_{l}, c_{n}\right), \\
& \beta=\beta\left(p_{i}, a_{j k}, b_{l}, c_{n}\right) .
\end{aligned}
$$

According to our initial hypothesis,

$$
\phi^{\prime}\left(p_{i}^{\prime}\right)=\int u\left(p_{i}^{\prime}, x_{i}^{\prime}\right) \psi^{\prime}\left(x_{i}^{\prime}\right) d x^{\prime} .
$$

Substituting from the transformation equations (5), (6) and from (9), we get

where

$$
\phi e^{\imath \beta}=\int u\left(\sum_{j} a_{i j} p_{j}+c_{i}, \sum_{j} a_{i j} x_{j}+b_{i}\right) \psi e^{\iota \alpha} d x,
$$

$$
\frac{\partial\left(x_{1}^{\prime}, x_{2}^{\prime}, x_{3}^{\prime}\right)}{\partial\left(x_{1}, x_{2}, x_{3}\right)}=\left|a_{i j}\right|= \pm 1 .
$$

The negative sign must be taken if the orthogonal transformation is indirect; in this case, it is necessary to replace $\beta$ by $\beta+\pi$ before arriving at equation (12).

It now follows from equations (3) and (12) that

$$
\int u\left(p_{i}, x_{i}\right) \psi\left(x_{i}\right) e^{i \beta} d x=\int u\left(\sum_{j} a_{i j} p_{j}+c_{i}, \sum_{j} a_{i j} x_{j}+b_{i}\right) \psi\left(x_{i}\right) e^{\iota \alpha} d x,
$$

since $\beta$ is independent of the $x_{i}$. This last equation is to be valid for arbitrary wave functions $\psi\left(x_{i}\right)$ which lead to convergent integrals. For this to be so, it is necessary that

$$
u\left(p_{i}, x_{i}\right) e^{\iota \beta}=u\left(\sum_{j} a_{i j} p_{j}+c_{i}, \sum_{j} a_{i j} x_{j}+b_{i}\right) e^{i \alpha}
$$

identically in the variables $x_{i}, p_{i}$ and parameters $a_{i j}, c_{i}, b_{i}$. The form of $u$ can now be deduced as follows: 
First, we note that

$$
\left|u\left(p_{i}, x_{i}\right)\right|=\left|u\left(\sum_{j} a_{i j} p_{j}+c_{i}, \sum_{j} a_{i j} x_{j}+b_{i}\right)\right| .
$$

Since $b_{i}, c_{i}$ are arbitrary, this identity implies that

$$
\left|u\left(p_{i}, x_{i}\right)\right|=\text { constant }=u_{0} .
$$

Taking arguments of both sides of the identity (15), we find

$$
\theta\left(p_{i}, x_{i}\right)+\beta=\theta\left(\sum_{j} a_{i j} p_{j}+c_{i}, \sum_{j} a_{i j} x_{j}+b_{i}\right)+\alpha,
$$

where

$$
\theta=\arg u \text {. }
$$

Differentiating equation (18) partially with respect of $p_{i}$ and $x_{j}$, since $\alpha$ is independent of $p_{i}$ and $\beta$ is independent of $x_{j}$, it follows that

$$
\frac{\partial^{2} \theta}{\partial p_{i} \partial x_{j}}=\sum_{r, s} \frac{\partial^{2} \theta}{\partial p_{r}^{\prime} \partial x_{s}^{\prime}} a_{r i} a_{s j} \text {. }
$$

The properties of the coefficients $a_{i j}$ enable us to write this relationship in the inverse form

$$
\frac{\partial^{2} \theta}{\partial p_{i}^{\prime} \partial x_{j}^{\prime}}=\sum_{r, s} a_{i r} a_{j s} \frac{\partial^{2} \theta}{\partial p_{r} \partial x_{s}},
$$

from which it is clear that the quantities $\partial^{2} \theta / \partial p_{i} \partial x_{j}$ transform between two rectangular cartesian frames like the elements of a tensor of the second rank.

Putting $x_{i}=0, p_{i}=0(i=1,2,3)$ in the identity $(21)$, we obtain

$$
\frac{\partial^{2} \theta}{\partial c_{i} \partial b_{j}}=\sum_{r, s} a_{i r} a_{i s}\left(\frac{\partial^{2} \theta}{\partial p_{r} \partial x_{s}}\right)_{0}
$$

where the subscript zero indicates that the arguments $p_{r}, x_{s}$ are put equal to zero after differentiation. But the right-hand member of equation (22) is independent of $c_{i}$ and $b_{j}$ and it follows, therefore, that the left-hand member is also. Thus

$$
\frac{\partial^{2} \theta}{\partial p_{i} \partial x_{j}}=\chi_{i j}
$$

where the $\chi_{i j}$ are constants. Equation (21) can now be written

$$
\chi_{i j}=\sum_{r, s} a_{i r} a_{j s} \chi_{r s}
$$

implying that $\chi_{i j}$ is a second rank tensor whose elements are the same in every frame. It is shown by $\mathrm{H}$. Jeffreys [3] that any such tensor must be an invariant multiple of the fundamental tensor, i.e. 


$$
\chi_{i j}=\gamma \delta_{i j} .
$$

We have proved, therefore, that

$$
\frac{\partial^{2} \theta}{\partial p_{i} \partial x_{j}}=\gamma \delta_{i j} .
$$

Integrating, we find that

$$
\theta=\gamma \sum_{i} p_{i} x_{i}+P+X,
$$

where $P$ is a function of the $p_{i}$ alone and $X$ is a function of the $x_{i}$ alone. Hence

$$
u=u_{0} \exp \left\{\iota\left(\gamma \sum_{i} p_{i} x_{i}+P+X\right)\right\}
$$

The transformation equations (3), (4) can now be written as

$$
\begin{aligned}
e^{-\iota P} \phi\left(p_{i}\right) & =\int u_{0} e^{\iota \gamma \sum p_{i} x_{i}} e^{\iota X} \psi\left(x_{i}\right) d x, \\
e^{\iota X} \psi\left(x_{i}\right) & =\int u_{0} e^{-\iota \gamma \sum p_{i} x_{i}} e^{-\iota P} \phi\left(p_{i}\right) d p .
\end{aligned}
$$

But the only connection between the physical world and the mathematical symbolism is that $|\psi|^{2},|\phi|^{2}$ are interpretable as probability densities. This connection is unaltered if we now absorb a factor $e^{\ell \boldsymbol{X}}$ in $\psi$ and a factor $e^{-\imath \boldsymbol{P}}$ in $\phi$, to yield new wave functions, which we can then again denote by $\psi, \phi$ respectively.

$\gamma$ is a fundamental constant of the physical world and its value can only be decided by comparing the physical implications of equations (29), (30), with actual observations. It is found that the agreement between theory and experiment can only be obtained if we take $\gamma=-1 / \hbar$ (the negative sign is purely conventional), where $2 \pi \hbar$ is the constant introduced originally by Planck. However, the most fundamental definition of Planck's constant is that it is the constant which arises at equation (25).

Finally, $u_{0}$ is determined by the requirement that the equations (29), (30) should be consistent; these equations are essentially those relating a function and its Fourier transform and it follows from the theory of this relationship that $u_{0}=h^{-\frac{3}{2}}$. Thus

$$
\begin{aligned}
\phi\left(p_{i}\right) & =h^{-\frac{3}{2}} \int e^{-i \Sigma p_{i} x_{i} / \hbar} \psi\left(x_{i}\right) d x, \\
\psi\left(x_{i}\right) & =h^{-\frac{3}{2}} \int e^{\imath \sum p_{i} x_{i} / \hbar} \phi\left(p_{i}\right) d p .
\end{aligned}
$$

Having established these transformation equations relating the coordinate and momentum representations of the state of a particle, the development of the theory of the quantum mechanics of a particle can proceed in a 
straightforward manner (see e.g. Tolman [2]). Thus, in the coordinate representation, it now follows directly that the operators representing the observables $x_{i}, p_{i}$ are $x_{i},-\imath \hbar \partial / \partial x_{i}$, and that, in the momentum representation, the corresponding operators are $\imath \hbar \partial / \partial p_{i}, p_{i}$.

\section{References}

[1] A. Landé, New Foundations of Quantum Mechanics (Cambridge University Press, 1965).

[2] R. C. Tolman, The Principles of Statistical Mechanics, Chap. VII (Oxford University Press, 1955).

[3] H. Jeffreys, Cartesian Tensors, Chap. VII (Cambridge University Press, 1957).

University of Canterbury

Christchurch, New Zealand 